TRANSACTIONS OF THE

AMERICAN MATHEMATICAL SOCIETY

Volume 350, Number 7, July 1998, Pages 2665-2685

S 0002-9947(98)02074-1

\title{
GENERATORS AND RELATIONS OF DIRECT PRODUCTS OF SEMIGROUPS
}

\author{
E. F. ROBERTSON, N. RUŠKUC, AND J. WIEGOLD
}

\begin{abstract}
The purpose of this paper is to give necessary and sufficient conditions for the direct product of two semigroups to be finitely generated, and also for the direct product to be finitely presented. As a consequence we construct a semigroup $S$ of order 11 such that $S \times T$ is finitely generated but not finitely presented for every finitely generated infinite semigroup $T$. By way of contrast we show that, if $S$ and $T$ belong to a wide class of semigroups, then $S \times T$ is finitely presented if and only if both $S$ and $T$ are finitely presented, exactly as in the case of groups and monoids.
\end{abstract}

\section{INTRODUCTION}

Given two groups (or, more generally, monoids) $G=\langle A \mid R\rangle$ and $H=\langle B \mid Q\rangle$, it is well known that their direct product $G \times H$ has a presentation

$$
\langle A, B \mid R, Q, a b=b a(a \in A, b \in B)\rangle .
$$

An immediate consequence of this is that $G \times H$ is finitely generated if and only if both $G$ and $H$ are finitely generated, and is finitely presented if and only if both $G$ and $H$ are finitely presented. By way of contrast, it is also well known that if $\mathbb{N}=\{1,2,3, \ldots\}$ is the additive semigroup of natural numbers, then although $\mathbb{N}$ is generated by a single element, $\mathbb{N} \times \mathbb{N}$ is not finitely generated. Indeed, any generating set for $\mathbb{N} \times \mathbb{N}$ must contain the set $\{(1, n): n \in \mathbb{N}\}$.

These observations naturally lead one to ask when, given two semigroups $S$ and $T$, the direct product $S \times T$ is finitely generated, and also when it is finitely presented. We solve both these problems in this paper.

In this context the case where both direct factors are finite is of no interest: the direct product is then finite and hence both finitely generated and finitely presented. In its main part the paper is concerned with direct products of two infinite semigroups. In Section 2 we give a necessary and sufficient condition for such a direct product to be finitely generated. In Section 3 we introduce some technical concepts related to semigroup presentations and state the main result of the paper, giving a necessary and sufficient condition for a direct product of two infinite semigroups to be finitely presented. Sections 4 and 5 contain the proof of this main theorem. In Section 6 we construct a semigroup $S$ which has the property that, for any finitely generated infinite semigroup $T$ satisfying $T^{2}=T$, the direct product $S \times T$ is finitely generated but not finitely presented. Even more surprisingly, in Section 8 we construct a semigroup $S$ with eleven elements such

Received by the editors September 24, 1996.

1991 Mathematics Subject Classification. Primary 20M05.

(C)1998 American Mathematical Society 
that $S \times T$ is finitely generated but not finitely presented for all finitely generated infinite semigroups $T$. By way of contrast, in Section 7 we prove that if $S$ and $T$ are finitely presented infinite semigroups belonging to a wide class of semigroups (which contains some well known subclasses, such as monoids and regular semigroups), then $S \times T$ is finitely presented. We conclude the paper by considering direct products $S \times T$ where $S$ is finite and $T$ is infinite.

We mention that finite presentability of some other semigroup constructions (such as wreath products and Rees matrix semigroups) was investigated in [10].

\section{Generators}

Let $S$ be a semigroup. An element $s \in S$ is said to be decomposable if there exist elements $s_{1}, s_{2} \in S$ such that $s=s_{1} s_{2}$. Thus the set of all decomposable elements of $S$ is

$$
S^{2}=S S=\left\{s_{1} s_{2}: s_{1}, s_{2} \in S\right\} .
$$

An element is indecomposable if it is not decomposable. The set of all indecomposable elements is $S \backslash S^{2}$. It is clear that this set is contained in every generating set for $S$.

The main result of this section is the following necessary and sufficient condition for a direct product to be finitely generated.

Theorem 2.1. Let $S$ and $T$ be two infinite semigroups. Then $S \times T$ is finitely generated if and only if both $S$ and $T$ are finitely generated and $S^{2}=S$ and $T^{2}=T$.

Proof. The theorem is an immediate consequence of Lemmas 2.2, 2.3 and Proposition 2.5 below.

Lemma 2.2. Let $S$ and $T$ be two semigroups. Denote by $\pi_{S}: S \times T \longrightarrow S$ the natural projection. If $A$ is a generating set for $S \times T$, then $\pi_{S}(A)$ is a generating set for $S$. In particular, if $S \times T$ is finitely generated then so is $S$.

Proof. The lemma follows from the fact that $\pi_{S}$ is an epimorphism.

Lemma 2.3. Let $S$ and $T$ be two semigroups. If $T$ is infinite and $S \times T$ is finitely generated, then $S^{2}=S$.

Proof. Assume that $S^{2} \neq S$, so that $S$ has an indecomposable element $s$. But then each of the infinitely many elements $(s, t)(t \in T)$ is indecomposable in $S \times T$, and hence it belongs to every generating set for $S \times T$, contradicting the assumption that $S \times T$ is finitely generated.

Lemma 2.4. Let $S$ be a semigroup such that $S^{2}=S$, and let $A=\left\{a_{i}: i \in I\right\}$ be a generating set for $S$. Then there exist elements $s_{i} \in S(i \in I)$ and a mapping $\zeta: I \longrightarrow I$ such that

$$
a_{i}=a_{\zeta(i)} s_{i}
$$

for all $i \in I$.

Proof. From $S^{2}=S$ it follows that $S$ has no indecomposable elements. Thus each $a_{i}$ can be written as a product $a_{i_{1}} a_{i_{2}} \ldots a_{i_{k}}$ of generators with $k \geq 2$. Now define $\zeta(i)=i_{1}$ and $s_{i}=a_{i_{2}} \ldots a_{i_{k}}$. 
Proposition 2.5. Let $S$ and $T$ be two semigroups satisfying $S^{2}=S$ and $T^{2}=T$. Let $A=\left\{a_{i}: i \in I\right\}$ and $B=\left\{b_{j}: j \in J\right\}$ be generating sets for $S$ and $T$ respectively. Choose elements $s_{i} \in S$ and $t_{j} \in S(i \in I, j \in J)$ and functions $\zeta: I \longrightarrow I$ and $\theta: J \longrightarrow J$ so that $a_{i}=a_{\zeta(i)} s_{i}$ for all $i \in I$ and $b_{j}=b_{\theta(j)} t_{j}$ for all $j \in J$. Then the set

$$
\left(A \cup\left\{s_{i}: i \in I\right\}\right) \times\left(B \cup\left\{t_{j}: j \in J\right\}\right)
$$

generates $S \times T$.

Proof. Let $s \in S$ be arbitrary, and assume that $s$ can be decomposed into a product of $m$ generators from $A$. By successively replacing an arbitrary generator $a_{i}$ by the product $a_{\zeta(i)} s_{i}$, we see that for every $n \geq m$ the element $s$ can be expressed as a product of $n$ elements from $A \cup\left\{s_{i}: i \in I\right\}$. Similarly, if an element $t \in T$ can be expressed as a product of $m$ generators from $B$, then for every $n \geq m$ it can be expressed as a product of $n$ elements from $B \cup\left\{t_{j}: j \in J\right\}$.

Now let $s \in S$ and $t \in T$ be arbitrary. Assume that $s$ can be written as a product of $m$ generators from $A$, and that $t$ can be written as a product of $n$ generators from $B$. Let $p=\max (m, n)$, and write $s$ and $t$ as products

$$
\begin{aligned}
& s=\alpha_{1} \alpha_{2} \ldots \alpha_{p}, \\
& t=\beta_{1} \beta_{2} \ldots \beta_{p}
\end{aligned}
$$

of $p$ elements from $A \cup\left\{s_{i}: i \in I\right\}$ and $B \cup\left\{t_{j}: j \in J\right\}$ respectively. Now we can write $(s, t)$ as a product of elements from

$$
\left(A \cup\left\{s_{i}: i \in I\right\}\right) \times\left(B \cup\left\{t_{j}: j \in J\right\}\right)
$$

as follows:

$$
(s, t)=\left(\alpha_{1}, \beta_{1}\right)\left(\alpha_{2}, \beta_{2}\right) \ldots\left(\alpha_{p}, \beta_{p}\right),
$$

and this completes the proof of the proposition.

Remark 2.6. If $S$ and $T$ have the property that $S^{2}=S$ and $T^{2}=T$, then we also have $(S \times T)^{2}=S \times T$. The converse is also true: if $(S \times T)^{2}=S \times T$ then $S^{2}=S$ and $T^{2}=T$, since $S$ and $T$ are homomorphic images of $S \times T$. This observation enables one to generalise Theorem 2.1 to arbitrary finite direct products $S_{1} \times S_{2} \times \ldots \times S_{k}$ of infinite semigroups. This direct product is finitely generated if and only if each $S_{i}(1 \leq i \leq k)$ is finitely generated and satisfies $S_{i}^{2}=S_{i}$.

The rank of a semigroup $S$, denoted by $\operatorname{rank}(S)$, is defined to be the minimal number of elements of a generating set of $S$. The standard generating set for the direct product $G \times H$ of two groups yields the following upper bound on the rank of $G \times H$ :

$$
\operatorname{rank}(G \times H) \leq \operatorname{rank}(G)+\operatorname{rank}(H) .
$$

(Actually, $\operatorname{rank}(G \times H)$ is often significantly smaller than $\operatorname{rank}(G)+\operatorname{rank}(H)$; see for example [7] and [26].) In the same way Proposition 2.5 yields an upper bound on the rank of the direct product of two infinite semigroups.

Corollary 2.7. Let $S$ and $T$ be two infinite semigroups such that $S^{2}=S$ and $T^{2}=T$. Then

$$
\operatorname{rank}(S \times T) \leq 4 \operatorname{rank}(S) \operatorname{rank}(T)
$$


Proof. If the generating sets $A$ and $B$ for $S$ and $T$ are chosen to have cardinalities $\operatorname{rank}(S)$ and $\operatorname{rank}(T)$ respectively, then the generating set for $S \times T$ established in Proposition 2.5 has the cardinality at most $4 \operatorname{rank}(S) \operatorname{rank}(T)$.

We do not know whether this upper bound can be attained. The following example, however, shows that the rank of the direct product can be significantly larger than the ranks of factors.

Example 2.8. Let $S$ be the semigroup free product of $m$ cyclic groups $\left\langle a_{i}\right\rangle(1 \leq$ $i \leq m$ ) of order 2 , and let $T$ be the semigroup free product of $n$ cyclic groups $\left\langle b_{j}\right\rangle$ $(1 \leq j \leq n)$ of order 2 . (For the definition of semigroup free products see [9], and for some combinatorial properties of this construction see [3] and [4].) Then clearly we have $S^{2}=S, T^{2}=T$, and

$$
\operatorname{rank}(S)=m, \operatorname{rank}(T)=n .
$$

Consider the direct product $S \times T$. Let $i$ and $j(1 \leq i \leq m, 1 \leq j \leq n)$ be arbitrary. The subsemigroup $P_{i j}=\left\langle a_{i}\right\rangle \times\left\langle b_{j}\right\rangle$ of $S \times T$ is a group isomorphic to the Klein four group. In particular

$$
\operatorname{rank}\left(P_{i j}\right)=2 .
$$

On the other hand the set $(S \times T) \backslash P_{i j}$ is an ideal of $S \times T$. Thus any generating set of $S \times T$ must contain a generating set for $P_{i j}$, and we conclude that

$$
\operatorname{rank}(S \times T) \geq 2 m n=2 \operatorname{rank}(S) \operatorname{rank}(T) .
$$

Actually, we have

$$
\operatorname{rank}(S \times T)=2 m n,
$$

since the set

$$
\left\{\left(a_{i}, b_{j}^{2}\right),\left(a_{i}^{2}, b_{j}\right): 1 \leq i \leq m, 1 \leq j \leq n\right\}
$$

is easily seen to generate $S \times T$.

We finish this section by establishing certain nice generating sets for semigroups $S$ satisfying $S^{2}=S$. These generating sets, in turn, yield nice generating sets for direct products, which we shall make use of in the following sections.

Definition 2.9. A generating set $A$ of a semigroup $S$ is said to be full if $A \subseteq A^{2}$, i.e. if every generator of $A$ can be expressed as a product of two generators of $A$.

Proposition 2.10. A semigroup $S$ has a full generating set $A$ if and only if $S^{2}=$ $S$. Furthermore, if $S$ is finitely generated, $A$ can be chosen to be finite.

Proof. $(\Rightarrow)$ If $A$ is a full generating set, then every element of $A$ is decomposable. Hence $S$ has no indecomposable elements, and so must satisfy $S^{2}=S$.

$(\Leftarrow)$ Assume that $S^{2}=S$, and let $A_{0}=\left\{a_{i}: i \in I\right\}$ be any generating set for $S$. Each $a_{i}(i \in I)$ is decomposable, and so we can write

$$
a_{i}=a_{\zeta(i, 1)} a_{\zeta(i, 2)} \ldots a_{\zeta\left(i, m_{i}\right)}
$$

where $m_{i} \geq 2$ and $\zeta(i, j) \in I$ for all $j\left(1 \leq j \leq m_{i}\right)$. For all $i$ and $j(i \in I$, $\left.1 \leq j \leq m_{i}-1\right)$ define

$$
\alpha_{i, j}=a_{\zeta(i, j+1)} \ldots a_{\zeta\left(i, m_{i}\right)}
$$

The set

$$
A=A_{0} \cup\left\{\alpha_{i, j}: i \in I, 1 \leq j \leq m_{i}-1\right\}
$$


is clearly a generating set for $S$. Also, from (1) and (2) it follows that

$$
\begin{aligned}
& a_{i}=a_{\zeta(i, 1)} \alpha_{i, 1}, \\
& \alpha_{i, j}=a_{\zeta(i, j+1)} \alpha_{i, j+1} \quad\left(1 \leq j \leq m_{i}-2\right), \\
& \alpha_{i, m_{i}-1}=a_{\zeta\left(i, m_{i}\right)}=a_{\zeta\left(\zeta\left(i, m_{i}\right), 1\right)} \alpha_{\zeta\left(i, m_{i}\right), 1} .
\end{aligned}
$$

Hence $A$ is full. Finally, we observe that if $A_{0}$ is finite, then so is $A$.

Corollary 2.11. Let $S$ and $T$ be two semigroups with $S^{2}=S$ and $T^{2}=T$, and let $A$ and $B$ be full generating sets for $S$ and $T$ respectively. Then the set $A \times B$ is a full generating set for $S \times T$. Moreover, if $S \times T$ is finitely generated, then the sets $A$ and $B$ can be chosen to be finite.

Proof. Since every generator from $A$ is a product of two generators, we can choose the elements $s_{i}(i \in I)$ in Proposition 2.5 so as to belong to $A$. Similarly, the elements $t_{j}(j \in J)$ can be chosen from $B$, and so the generating set from Proposition 2.5 is $A \times B$. Next, from

$$
A \times B \subseteq A^{2} \times B^{2}=(A \times B)^{2}
$$

it follows that $A \times B$ is full. Finally, if $S \times T$ is finitely generated, it follows that both $S$ and $T$ are finitely generated by Lemma 2.2 , and hence $A$ and $B$ can be chosen to be finite by Proposition 2.10 .

We finish this section by recording a property of semigroups with full generating sets that we will need in the following sections.

Lemma 2.12. Let $S$ be a semigroup and let $A$ be a full generating set for $S$. If an element $s \in S$ can be expressed as a product of $m$ generators from $A$, then for every $n \geq m$ it can also be expressed as a product of $n$ generators from $A$.

Proof. The assertion is an immediate consequence of the fact that any generator from $A$ can be replaced by a product of two generators from $A$.

\section{Presentations, stability and the main Result}

Let $A$ be an alphabet. By $A^{+}$we denote the set of all (non-empty) words over $A$. The length of a word $w \in A^{+}$is denoted by $|w|$. The set $A^{+}$is a semigroup with concatenation of words as multiplication. This semigroup is free on $A$. A presentation is a pair $\mathfrak{P}=\langle A \mid R\rangle$, where $R \subseteq A^{+} \times A^{+}$is a set of pairs of words. A pair $(u, v) \in R$ is usually written as $u=v$ and is called a defining relation. A semigroup $S$ is said to be defined by the presentation $\mathfrak{P}$ if $S \cong A^{+} / R^{\sharp}$, where $R^{\sharp}$ is the smallest congruence on $A^{+}$containing $R$. Intuitively, $S$ is the largest semigroup generated by $A$ in which the generators satisfy the relations from $R$. Usually we identify $S$ with $A^{+} / R^{\sharp}$. Thus the elements of $S$ are congruence classes $w / R^{\sharp}$ of words $w \in A^{+}$. To put it differently, every word $w \in A^{+}$represents an element of $S$. Often we identify a word with the element it represents. To lessen the likelihood of confusion in doing this, we introduce the following convention. For two words $w_{1}, w_{2} \in A^{+}$we write $w_{1} \equiv w_{2}$ if they are equal in $A^{+}$(i.e. if they are identical as words), and we write $w_{1}=w_{2}$ if they represent the same element of $S$ (i.e. if $\left.\left(w_{1}, w_{2}\right) \in R^{\sharp}\right)$.

Let us denote the empty word by $\epsilon$, and let $A^{*}=A^{+} \cup\{\epsilon\}$. For two words $w_{1}, w_{2} \in A^{+}$we say that $w_{2}$ is obtained from $w_{1}$ by one application of one relation from $R$ if we can write $w_{1} \equiv \alpha u \beta$ and $w_{2} \equiv \alpha v \beta$, where $(u=v) \in R$ or $(v=u) \in R$ 
and $\alpha, \beta \in A^{*}$. An elementary sequence (from $w_{1}$ to $w_{2}$ ) with respect to $\mathfrak{P}$ is a sequence

$$
w_{1} \equiv \alpha_{1}, \alpha_{2}, \ldots, \alpha_{k} \equiv w_{2},
$$

of words from $A^{+}$, such that for each $i(1 \leq i \leq k-1)$ either $\alpha_{i} \equiv \alpha_{i+1}$ or $\alpha_{i+1}$ is obtained from $\alpha_{i}$ by one application of one relation from $R$. If, for two words $w_{1}, w_{2} \in A^{+}$, such a sequence exists, we say that the relation $w_{1}=w_{2}$ is a consequence of $\mathfrak{P}$ (alternatively, of $R$ ). In what follows we shall use frequently the following basic

Proposition 3.1. Let $\mathfrak{P}=\langle A \mid R\rangle$ be a presentation, let $S$ be the semigroup defined by it, and let $w_{1}, w_{2} \in A^{+}$be arbitrary words. Then the relation $w_{1}=w_{2}$ holds in $S$ if and only if it is a consequence of $\mathfrak{P}$.

A semigroup $S$ is said to be finitely presented if it can be defined by a finite presentation, i.e. by a presentation $\langle A \mid R\rangle$ in which both $A$ and $R$ are finite. It is a well known fact that if a semigroup can be defined by a finite presentation with respect to one finite generating set then it can be defined by a finite presentation with respect to any other finite generating set.

The aim of this section is to state a necessary and sufficient condition for the direct product of two infinite semigroups to be finitely presented, which we will then prove in the following two sections. In order to do this we first need to define some technical properties of presentations.

Definition 3.2. Let $\mathfrak{P}=\langle A \mid R\rangle$ be a presentation, let $S$ be the semigroup defined by it, and let $w_{1}, w_{2} \in A^{+}$be arbitrary words. The pair $\left(w_{1}, w_{2}\right)$ is called a critical pair (for $S$ with respect to $\mathfrak{P}$ ) if the following conditions are satisfied:

(i) the relation $w_{1}=w_{2}$ holds in $S$;

(ii) for every elementary sequence $w_{1} \equiv \alpha_{1}, \alpha_{2}, \ldots, \alpha_{k} \equiv w_{2}$ from $w_{1}$ to $w_{2}$ with respect to $\mathfrak{P}$ there exists $i(1 \leq i \leq k)$ such that $\left|\alpha_{i}\right|<\min \left(\left|w_{1}\right|,\left|w_{2}\right|\right)$.

Definition 3.3. Let $S$ be a semigroup with a finite generating set $A$. We say that $S$ is stable (with respect to $A$ ) if there exists a finite presentation $\mathfrak{P}=\langle A \mid R\rangle$, defining $S$ in terms of $A$, with respect to which $S$ has no critical pairs.

Stability is invariant under the change of the (finite) generating set, as the following proposition shows.

Proposition 3.4. Let $S$ be a semigroup, and let $A=\left\{a_{i}: i \in I\right\}$ and $B=\left\{b_{j}\right.$ : $j \in J\}$ be two finite generating sets for $S$. If $S$ is stable with respect to $A$, then it is stable with respect to $B$ as well.

Proof. Let $\mathfrak{P}=\langle A \mid R\rangle$ be a finite presentation, defining $S$ in terms of $A$, with respect to which $S$ has no critical pairs. Since $B$ is a generating set for $S$, each $a_{i}(i \in I)$ can be expressed as a product of generators from $B$. Thus there exist words $\alpha_{i} \in B^{+}(i \in I)$ such that $a_{i}=\alpha_{i}$ in $S$. Let $\phi: A^{+} \longrightarrow B^{+}$be the unique homomorphism extending the mapping $a_{i} \mapsto \alpha_{i}(i \in I)$. Similarly, choose words $\beta_{j} \in A^{+}(j \in J)$ such that $b_{j}=\beta_{j}$ in $S$, and let $\psi: B^{+} \longrightarrow A^{+}$be the unique homomorphism extending the mapping $b_{j} \mapsto \beta_{j}(j \in J)$.

Now it is easy to show by using semigroup Tietze transformations (or directly) that the presentation

$$
\mathfrak{Q}=\left\langle B \mid \phi(R), b_{j}=\phi\left(\psi\left(b_{j}\right)\right)(j \in J)\right\rangle
$$


defines $S$ in terms of $B$. (For the definitions of Tietze transformations see [14], and for their semigroup variants see [16], [19], [20] or [23]. In the above presentation $\phi(R)$ denotes the set $\{\phi(u)=\phi(v):(u=v) \in R\}$.) We shall show that $S$ has no critical pairs with respect to $\mathfrak{Q}$.

Let $w_{1}, w_{2} \in B^{+}$be any two words such that $w_{1}=w_{2}$ in $S$. Then the relation $\psi\left(w_{1}\right)=\psi\left(w_{2}\right)$ also holds in $S$. Since $S$ has no critical pairs with respect to $\mathfrak{P}$, it follows that there exists an elementary sequence

$$
\psi\left(w_{1}\right) \equiv \gamma_{1}, \gamma_{2}, \ldots, \gamma_{m} \equiv \psi\left(w_{2}\right)
$$

with respect to $\mathfrak{P}$, such that $\left|\gamma_{k}\right| \geq \min \left(\left|\psi\left(w_{1}\right)\right|,\left|\psi\left(w_{2}\right)\right|\right)$ for all $k(1 \leq k \leq m)$. But then the sequence

$$
\phi\left(\psi\left(w_{1}\right)\right) \equiv \phi\left(\gamma_{1}\right), \phi\left(\gamma_{2}\right), \ldots, \phi\left(\gamma_{m}\right) \equiv \phi\left(\psi\left(w_{2}\right)\right)
$$

is elementary with respect to $\mathfrak{Q}$, and we also have

$$
\left|\phi\left(\gamma_{k}\right)\right| \geq\left|\gamma_{k}\right| \geq \min \left(\left|\psi\left(w_{1}\right)\right|,\left|\psi\left(w_{2}\right)\right|\right) \geq \min \left(\left|w_{1}\right|,\left|w_{2}\right|\right) .
$$

Also, by successively replacing every $b_{j}(j \in J)$ in $w_{1}$ and $w_{2}$ by $\phi\left(\psi\left(b_{j}\right)\right)$, one constructs 'increasing' elementary sequences

$$
\begin{aligned}
& w_{1} \equiv \gamma_{1}^{\prime}, \gamma_{2}^{\prime}, \ldots, \gamma_{n}^{\prime} \equiv \phi\left(\psi\left(w_{1}\right)\right), \\
& w_{2} \equiv \gamma_{1}^{\prime \prime}, \gamma_{2}^{\prime \prime}, \ldots, \gamma_{p}^{\prime \prime} \equiv \phi\left(\psi\left(w_{2}\right)\right),
\end{aligned}
$$

such that $\left|\gamma_{k}^{\prime}\right| \geq\left|w_{1}\right|(1 \leq k \leq n)$ and $\left|\gamma_{k}^{\prime \prime}\right| \geq\left|w_{2}\right|(1 \leq k \leq p)$. By combining sequences (3), (4) and (5) we obtain an elementary sequence from $w_{1}$ to $w_{2}$ with respect to $\mathfrak{Q}$ in which no term is shorter than $\min \left(\left|w_{1}\right|,\left|w_{2}\right|\right)$. This completes the proof of the proposition.

Now we can state the main result of this paper.

Theorem 3.5. Let $S$ and $T$ be two infinite semigroups. The direct product $S \times T$ is finitely presented if and only if the following conditions are satisfied:

(i) $S^{2}=S$ and $T^{2}=T$;

(ii) $S$ and $T$ are (finitely presented and) stable.

Proof. If $S \times T$ is finitely presented, then it is also finitely generated. Hence $S$ and $T$ must be finitely generated and satisfy $S^{2}=S$ and $T^{2}=T$ by Theorem 2.1. That $S$ and $T$ must also be stable follows from Proposition 4.1 below. The converse statement follows from Proposition 5.7 in Section 5.

\section{Stability is NECESSARY}

In this section we give the result needed for the proof of the direct implication of Theorem 3.5.

Proposition 4.1. Let $S$ and $T$ be two semigroups satisfying $S^{2}=S$ and $T^{2}=T$. Let $A=\left\{a_{i}: i \in I\right\}$ and $B=\left\{b_{j}: j \in J\right\}$ be full generating sets for $S$ and $T$ respectively, and let $\mathfrak{P}=\langle A \times B \mid R\rangle$ be a presentation for $S \times T$ in terms of the generating set $A \times B$. Denote by $\pi_{A}:(A \times B)^{+} \longrightarrow A^{+}$the unique homomorphism extending the mapping $\left(a_{i}, b_{j}\right) \mapsto a_{i}(i \in I, j \in J)$. Then $S$ is defined by the presentation

$$
\mathfrak{Q}=\left\langle A \mid \pi_{A}(R)\right\rangle .
$$

Furthermore, if $T$ is infinite, $S$ has no critical pairs with respect to $\mathfrak{Q}$. So, in this case, if $S \times T$ is finitely presented then $S$ is stable. 
Proof. Note that $S$ and $T$ have full generating sets by Proposition 2.10, and that the set $A \times B$ generates $S \times T$ by Corollary 2.11. Also, the final statement of the proposition follows easily from the first two. Indeed, assume that $S \times T$ is finitely presented. Then $S$ and $T$ are finitely generated by Lemma 2.2, and so $A$ and $B$ can be chosen to be finite by Proposition 2.10. Now $S \times T$ can be defined by a finite presentation $\langle A \times B \mid R\rangle$ in terms of the generating set $A \times B$. By the first two statements of the proposition, $S$ is defined by the finite presentation $\left\langle A \mid \pi_{A}(R)\right\rangle$ and has no critical pairs with respect to it. Hence $S$ is stable.

Now we prove that $\mathfrak{Q}$ is a presentation for $S$. We do this by showing that all the relations of $\mathfrak{Q}$ hold in $S$, and that every relation holding in $S$ is a consequence of $\mathfrak{Q}$. First, however, we introduce some more notation.

By $\phi$ we denote the unique homomorphism $(A \times B)^{+} \longrightarrow S \times T$ extending the inclusion mapping $A \times B \hookrightarrow S \times T$, and by $\phi_{S}$ we denote the unique homomorphism $A^{+} \longrightarrow S$ extending the inclusion mapping $A \hookrightarrow S$. Also we let $\pi_{S}$ denote the natural projection $S \times T \longrightarrow S$. It is easy to see that the diagram

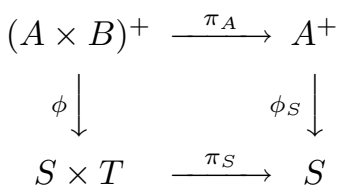

commutes.

To show that an arbitrary relation $\pi_{A}(u)=\pi_{A}(v)$ of $\mathfrak{Q}$ holds in $S$ we have to show that applying $\phi_{S}$ to both sides of that relation yields the same element of $S$. Since the relation $u=v$ holds in $S \times T$ we have $\phi(u)=\phi(v)$ in $S \times T$, and hence

$$
\phi_{S} \pi_{A}(u)=\pi_{S} \phi(u)=\pi_{S} \phi(v)=\phi_{S} \pi_{A}(v),
$$

as required.

Now let $w_{1}, w_{2} \in A^{+}$be any two words such that the relation $w_{1}=w_{2}$ holds in $S$. We want to show that the relation $w_{1}=w_{2}$ is a consequence of $\mathfrak{Q}$. If

$$
\begin{aligned}
& w_{1} \equiv a_{i_{1}} a_{i_{2}} \ldots a_{i_{m}}, \\
& w_{2} \equiv a_{k_{1}} a_{k_{2}} \ldots a_{k_{n}},
\end{aligned}
$$

we claim that there exist $b_{j_{1}}, \ldots, b_{j_{m}}, b_{l_{1}}, \ldots, b_{l_{n}} \in B$ such that the relation

$$
\left(a_{i_{1}}, b_{j_{1}}\right)\left(a_{i_{2}}, b_{j_{2}}\right) \ldots\left(a_{i_{m}}, b_{j_{m}}\right)=\left(a_{k_{1}}, b_{l_{1}}\right)\left(a_{k_{2}}, b_{l_{2}}\right) \ldots\left(a_{k_{n}}, b_{l_{n}}\right)
$$

holds in $S \times T$. Without loss of generality we may assume that $m \leq n$. Let us choose $b_{j_{1}}, \ldots, b_{j_{m}}$ arbitrarily. By Corollary $2.11 A \times B$ is a full generating set for $S \times T$. Hence, by Lemma 2.12 , the word $\left(a_{i_{1}}, b_{j_{1}}\right)\left(a_{i_{2}}, b_{j_{2}}\right) \ldots\left(a_{i_{m}}, b_{j_{m}}\right)$ is equal in $S \times T$ to a word of length $n$ :

$$
\left(a_{i_{1}}, b_{j_{1}}\right)\left(a_{i_{2}}, b_{j_{2}}\right) \ldots\left(a_{i_{m}}, b_{j_{m}}\right)=\left(a_{k_{1}^{\prime}}, b_{l_{1}}\right)\left(a_{k_{2}^{\prime}}, b_{l_{2}}\right) \ldots\left(a_{k_{n}^{\prime}}, b_{l_{n}}\right) .
$$

Since in $S$ we have

$$
a_{k_{1}^{\prime}} a_{k_{2}^{\prime}} \ldots a_{k_{n}^{\prime}}=a_{i_{1}} a_{i_{2}} \ldots a_{i_{m}} \equiv w_{1}=w_{2} \equiv a_{k_{1}} a_{k_{2}} \ldots a_{k_{n}},
$$

it follows that in $S \times T$ we have

$$
\left(a_{k_{1}^{\prime}}, b_{l_{1}}\right)\left(a_{k_{2}^{\prime}}, b_{l_{2}}\right) \ldots\left(a_{k_{n}^{\prime}}, b_{l_{n}}\right)=\left(a_{k_{1}}, b_{l_{1}}\right)\left(a_{k_{2}}, b_{l_{2}}\right) \ldots\left(a_{k_{n}}, b_{l_{n}}\right) .
$$

By combining (7) and (8) we conclude that relation (6) holds in $S \times T$, as claimed. 
Denote by $w_{3}$ and $w_{4}$ the left hand side and the right hand side respectively of relation (6). Since $w_{3}=w_{4}$ holds in $S \times T$, and since $\mathfrak{P}$ is a presentation for $S \times T$, there exists an elementary sequence

$$
w_{3} \equiv \alpha_{1}, \alpha_{2}, \ldots, \alpha_{p} \equiv w_{4}
$$

with respect to $\mathfrak{P}$. We claim that

$$
w_{1} \equiv \pi_{A}\left(w_{3}\right) \equiv \pi_{A}\left(\alpha_{1}\right), \pi_{A}\left(\alpha_{2}\right), \ldots, \pi_{A}\left(\alpha_{p}\right) \equiv \pi_{A}\left(w_{4}\right) \equiv w_{2}
$$

is an elementary sequence with respect to $\mathfrak{Q}$. Indeed, if $\alpha_{i} \equiv \alpha_{i+1}$ then $\pi_{A}\left(\alpha_{i}\right) \equiv$ $\pi_{A}\left(\alpha_{i+1}\right)$, while if $\alpha_{i} \equiv \beta u \gamma$ and $\alpha_{i+1} \equiv \beta v \gamma$, with $(u=v) \in R$ and $\beta, \gamma \in$ $(A \times B)^{*}$, then $\pi_{A}\left(\alpha_{i}\right) \equiv \pi_{A}(\beta) \pi_{A}(u) \pi_{A}(\gamma), \pi_{A}\left(\alpha_{i+1}\right) \equiv \pi_{A}(\beta) \pi_{A}(v) \pi_{A}(\gamma)$ and $\left(\pi_{A}(u)=\pi_{A}(v)\right) \in \pi_{A}(R)$. We conclude that the relation $w_{1}=w_{2}$ is a consequence of $\mathfrak{Q}$ as required, and this completes the proof of the fact that $\mathfrak{Q}$ is a presentation for $S$.

Now we prove that $S$ has no critical pairs with respect to $\mathfrak{Q}$, under the assumption that $T$ is infinite. We will do this by showing that in this case the elementary sequence (10) can be chosen so as to contain no term shorter than $\min \left(\left|w_{1}\right|,\left|w_{2}\right|\right)$. Recall that, in the above proof that $\mathfrak{Q}$ is a presentation for $S$, the elements $b_{j_{1}}, \ldots, b_{j_{m}}$ have been chosen arbitrarily. This time we choose them so that the word

$$
w_{5} \equiv b_{j_{1}} \ldots b_{j_{m}}
$$

is not equal in $T$ to a shorter word: this can be done for any $m$ since $T$ is infinite. Let

$$
w_{6} \equiv b_{l_{1}} \ldots b_{l_{n}},
$$

and let $\pi_{B}:(A \times B)^{+} \longrightarrow B^{+}$be the unique homomorphism extending the mapping $\left(a_{i}, b_{j}\right) \mapsto b_{j}(i \in I, j \in J)$. By applying $\pi_{B}$ to the elementary sequence (9) we obtain the following relations:

$$
w_{5} \equiv \pi_{B}\left(\alpha_{1}\right)=\pi_{B}\left(\alpha_{2}\right)=\ldots=\pi_{B}\left(\alpha_{p}\right) \equiv w_{6},
$$

holding in $T$. By the choice of $w_{5}$ we have

$$
\left|\pi_{B}\left(\alpha_{l}\right)\right| \geq\left|w_{5}\right|=m(1 \leq l \leq p) .
$$

On the other hand we have

$$
\left|\pi_{B}\left(\alpha_{l}\right)\right|=\left|\alpha_{l}\right|=\left|\pi_{A}\left(\alpha_{l}\right)\right| \quad(1 \leq l \leq p),
$$

so that we conclude that

$$
\left|\pi_{A}\left(\alpha_{l}\right)\right| \geq m=\min \left(\left|w_{1}\right|,\left|w_{2}\right|\right) \quad(1 \leq l \leq p) .
$$

Therefore $\left(w_{1}, w_{2}\right)$ is not a critical pair, and this completes the proof of the proposition.

\section{UNIFORMITY AND STABILITY}

In order to prove the converse part of Theorem 3.5 we find a presentation for $S \times T$, given presentations for $S$ and $T$ and assuming that both $S$ and $T$ are stable and satisfy $S^{2}=S$ and $T^{2}=T$.

We start working towards this goal by showing that every semigroup $S$ with $S^{2}=S$ can be defined by a presentation of a specific form. 
Definition 5.1. Let $A=\left\{a_{i}: i \in I\right\}$ be an alphabet. A presentation $\mathfrak{P}$ over $A$ is uniform if it has the form

$$
\mathfrak{P}=\left\langle A \mid a_{i}=a_{\zeta(i)} a_{\eta(i)}, R(i \in I)\right\rangle,
$$

where $\zeta, \eta: I \longrightarrow I$ are mappings, and $R$ is a set of relations such that for every relation $(u=v) \in R$ we have $|u|=|v|$.

Obviously, if a semigroup $S$ is defined by a uniform presentation in terms of a generating set $A$, then $A$ is a full generating set for $S$. By Lemma 2.12 every word in $A^{+}$of length $m$ is equal in $S$ to a word of length $n$ for every $n \geq m$. The following definition makes this more formal.

Definition 5.2. Let $A=\left\{a_{i}: i \in I\right\}$ be an alphabet, and let $\zeta, \eta: I \longrightarrow I$ be two mappings. Denote by $\mathbb{N}_{0}=\{0,1,2, \ldots\}$ the set of non-negative integers. The extension mapping (associated to $\zeta$ and $\eta$ ) is the mapping $\lambda: A^{+} \times \mathbb{N}_{0} \longrightarrow A^{+}$ defined by

$$
\begin{aligned}
& \lambda\left(w^{\prime} a_{i}, n\right) \equiv w^{\prime} a_{\zeta(i)} a_{\zeta \eta(i)} a_{\zeta \eta^{2}(i)} \ldots a_{\zeta \eta^{n-1}(i)} a_{\eta^{n}(i)}, \\
& \lambda(w, 0) \equiv w
\end{aligned}
$$

for $w \equiv w^{\prime} a_{i} \in A^{+}$and $n>0$.

Typically, the mappings $\zeta$ and $\eta$ come from a uniform presentation. In the following lemma we give the basic properties of extension mappings arising in this way.

Lemma 5.3. Let

$$
\mathfrak{P}=\left\langle A \mid a_{i}=a_{\zeta(i)} a_{\eta(i)}, R(i \in I)\right\rangle
$$

be a uniform presentation, let $S$ be the semigroup defined by it, and let $\lambda$ be the associated extension mapping. For any $w \in A^{+}$and any $n \in \mathbb{N}_{0}$ the following statements hold:

(i) the relation $\lambda(w, n)=w$ holds in $S$;

(ii) $|\lambda(w, n)|=|w|+n$.

Proof. The assertions follow from the fact that $\lambda(w, n)$ is obtained from $w$ by $n$ applications of relations of the form $a_{i}=a_{\zeta(i)} a_{\eta(i)}$.

Definition 5.4. Let

$$
\mathfrak{P}=\left\langle A \mid a_{i}=a_{\zeta(i)} a_{\eta(i)}, R(i \in I)\right\rangle
$$

be a uniform presentation, and let $\lambda$ be the associated extension mapping. The uniformity mapping is the mapping

$$
\nu: A^{+} \times A^{+} \longrightarrow\left\{\left(w_{1}, w_{2}\right) \in A^{+} \times A^{+}:\left|w_{1}\right|=\left|w_{2}\right|\right\}
$$

defined by

$$
\nu(u, v)= \begin{cases}(u, v) & \text { if }|u|=|v|, \\ (\lambda(u,|v|-|u|), v) & \text { if }|u|<|v|, \\ (u, \lambda(v,|u|-|v|)) & \text { if }|u|>|v| .\end{cases}
$$

If the pair $(u, v)$ is actually a relation, then we shall interpret $\nu(u, v)$ as a relation as well.

The following proposition shows how uniformity relates to some other concepts introduced earlier in this paper. 
Proposition 5.5. A semigroup $S$ can be defined by a uniform presentation if and only if $S^{2}=S$. Furthermore, if $S$ is finitely presented then it can be defined by a finite uniform presentation, and if $S$ is stable then it has no critical pairs with respect to a finite uniform presentation.

Proof. $(\Rightarrow)$ If $S$ can be defined by a uniform presentation in terms of a generating set $A$, then, because of the relations $a_{i}=a_{\zeta(i)} a_{\eta(i)}, A$ is full, and hence $S^{2}=S$ by Proposition 2.10.

$(\Leftarrow)$ Assume that $S^{2}=S$. Then $S$ has a full generating set $A=\left\{a_{i}: i \in I\right\}$ by Proposition 2.10. Thus $A \subseteq A^{2}$, and we can write

$$
a_{i}=a_{\zeta(i)} a_{\eta(i)} \quad(i \in I) .
$$

Let $\lambda$ and $\nu$ be respectively the extension mapping and the uniformity mapping associated to $\zeta$ and $\eta$.

Let $\mathfrak{P}=\langle A \mid R\rangle$ be any presentation for $S$ in terms of $A$. Note that any word $w \in A^{+}$can be transformed into the word $\lambda(w, n)\left(n \in \mathbb{N}_{0}\right)$ by using relations (11). Thus the presentation

$$
\overline{\mathfrak{P}}=\left\langle A \mid a_{i}=a_{\zeta(i)} a_{\eta(i)}, \bar{R}\right\rangle,
$$

with

$$
\bar{R}=\{\nu(u=v):(u=v) \in R\},
$$

is equivalent to $\mathfrak{P}$, and so it defines $S$. This presentation is also obviously uniform, and is finite if $\mathfrak{P}$ is finite.

To complete the proof of the proposition we assume that $S$ has no critical pairs with respect to $\mathfrak{P}$, and then prove that it has no critical pairs with respect to $\overline{\mathfrak{P}}$. Let $w_{1}, w_{2} \in A^{+}$be any two words such that the relation $w_{1}=w_{2}$ holds in $S$. By the assumption there exists an elementary sequence

$$
w_{1} \equiv \alpha_{1}, \alpha_{2}, \ldots, \alpha_{m} \equiv w_{2}
$$

with respect to $\mathfrak{P}$, such that $\left|\alpha_{k}\right| \geq \min \left(\left|w_{1}\right|,\left|w_{2}\right|\right)$ for all $k(1 \leq k \leq m)$.

Let $k(1 \leq k<m)$ be arbitrary. We claim that there exists an elementary sequence from $\alpha_{k}$ to $\alpha_{k+1}$ with respect to $\overline{\mathfrak{P}}$ in which no term is shorter than $\min \left(\left|\alpha_{k}\right|,\left|\alpha_{k+1}\right|\right)$. By assembling all these elementary sequences together we obtain an elementary sequence from $w_{1}$ to $w_{2}$ with respect to $\overline{\mathfrak{P}}$ in which no term is shorter than $\min \left(\left|w_{1}\right|,\left|w_{2}\right|\right)$. Thus we conclude that $S$ has no critical pairs with respect to $\overline{\mathfrak{P}}$, as required.

Now we prove the claim. If $\alpha_{k} \equiv \alpha_{k+1}$ there is nothing to prove. Otherwise we can write

$$
\alpha_{k} \equiv \beta u \gamma, \alpha_{k+1} \equiv \beta v \gamma,
$$

where $(u=v) \in R$ and $\beta, \gamma \in A^{*}$. If $|u|=|v|$ then $\nu(u=v)$ is $u=v$, and it belongs to $\bar{R}$. Let us now consider the case $|u|<|v|$, and let $n=|v|-|u|$. If $u \equiv u^{\prime} a_{i}$, then the sequence

$$
\begin{aligned}
& \alpha_{k} \equiv \beta u \gamma \equiv \beta u^{\prime} a_{i} \gamma, \beta u^{\prime} a_{\zeta(i)} a_{\eta(i)} \gamma, \beta u^{\prime} a_{\zeta(i)} a_{\zeta \eta(i)} a_{\eta^{2}(i)} \gamma, \ldots \\
& \beta u^{\prime} a_{\zeta(i)} a_{\zeta \eta(i)} \ldots a_{\zeta \eta^{n-1}(i)} a_{\eta^{n}(i)} \gamma \equiv \beta \lambda(u, n) \gamma, \beta v \gamma \equiv \alpha_{k+1}
\end{aligned}
$$

is elementary with respect to $\overline{\mathfrak{P}}$. Moreover, the terms of the sequence have increasing lengths, and so no term is shorter than $\alpha_{k}$. The case $|u|>|v|$ is dealt 
with similarly, and this completes the proof of the claim, as well as that of the proposition.

We need one more technical device before we can state our result.

Definition 5.6. Let $A=\left\{a_{i}: i \in I\right\}$ and $B=\left\{b_{j}: j \in J\right\}$ be two alphabets. The decomposition mapping is the mapping

$$
\xi:\left\{\left(w_{1}, w_{2}\right) \in A^{+} \times B^{+}:\left|w_{1}\right|=\left|w_{2}\right|\right\} \longrightarrow(A \times B)^{+}
$$

defined by

$$
\xi\left(a_{i_{1}} a_{i_{2}} \ldots a_{i_{n}}, b_{j_{1}} b_{j_{2}} \ldots b_{j_{n}}\right) \equiv\left(a_{i_{1}}, b_{j_{1}}\right)\left(a_{i_{2}}, b_{j_{2}}\right) \ldots\left(a_{i_{n}}, b_{j_{n}}\right) .
$$

Proposition 5.7. Let $S$ and $T$ be two stable semigroups satisfying $S^{2}=S$ and $T^{2}=T$. Let $A=\left\{a_{i}: i \in I\right\}$ and $B=\left\{b_{j}: j \in J\right\}$ be finite full generating sets for $S$ and $T$ respectively, and let

$$
\begin{aligned}
& \mathfrak{P}=\left\langle A \mid a_{i}=a_{\zeta(i)} a_{\eta(i)}, R \quad(i \in I)\right\rangle, \\
& \mathfrak{Q}=\left\langle B \mid b_{j}=b_{\theta(j)} b_{\iota(j)}, Q \quad(j \in J)\right\rangle,
\end{aligned}
$$

be finite uniform presentations for $S$ and $T$ respectively, with respect to which $S$ and $T$ have no critical pairs. If $\xi$ denotes the decomposition mapping, then the direct product $S \times T$ is defined by the presentation

$$
\begin{aligned}
\mathfrak{R}=\langle A \times B \quad| & \xi\left(u_{1}, \alpha\right)=\xi\left(v_{1}, \alpha\right), \\
& \xi\left(\beta, u_{2}\right)=\xi\left(\beta, v_{2}\right), \\
& \xi\left(a_{i_{1}} a_{\zeta\left(i_{2}\right)} a_{\eta\left(i_{2}\right)}, \gamma\right)=\xi\left(a_{\zeta\left(i_{1}\right)} a_{\eta\left(i_{1}\right)} a_{i_{2}}, \gamma\right), \\
& \xi\left(\delta, b_{j_{1}} b_{\theta\left(j_{2}\right)} b_{\iota\left(j_{2}\right)}\right)=\xi\left(\delta, b_{\theta\left(j_{1}\right)} b_{\iota\left(j_{1}\right)} b_{j_{2}}\right), \\
& \left(a_{i}, b_{j}\right)=\left(a_{\zeta(i)}, b_{\theta(j)}\right)\left(a_{\eta(i)}, b_{\iota(j)}\right) \\
& \left(\left(u_{1}=v_{1}\right) \in R ; \alpha \in B^{+} ;\left|u_{1}\right|=|\alpha| ;\left(u_{2}=v_{2}\right) \in Q ;\right. \\
& \beta \in A^{+} ;\left|u_{2}\right|=|\beta| ; i_{1}, i_{2} \in I ; \gamma \in B^{+} ;|\gamma|=3 ; \\
& \left.\left.j_{1}, j_{2} \in J ; \delta \in A^{+} ;|\delta|=3 ; i \in I ; j \in J\right)\right\rangle .
\end{aligned}
$$

In particular, $S \times T$ is finitely presented.

In order to prove Proposition 5.7 we need some more technical results. The notation will remain the same as in the proposition.

Lemma 5.8. Let $\alpha, \gamma \in A^{+}$and $\beta, \delta \in B^{+}$be arbitrary words such that $|\alpha|=|\beta|$ and $|\gamma|=|\delta|$. Then

$$
\xi(\alpha \gamma, \beta \delta) \equiv \xi(\alpha, \beta) \xi(\gamma, \delta) .
$$

Proof. The assertion follows immediately from Definition 5.6.

Lemma 5.9. (i) Let $\alpha_{1} a_{i} \alpha_{2} a_{k} \alpha_{3} \in A^{+}$be an arbitrary word of length $m-1 \geq 2$, and let $\beta \in B^{+}$be an arbitrary word of length $m$. Then the relation

$$
\xi\left(\alpha_{1} a_{\zeta(i)} a_{\eta(i)} \alpha_{2} a_{k} \alpha_{3}, \beta\right)=\xi\left(\alpha_{1} a_{i} \alpha_{2} a_{\zeta(k)} a_{\eta(k)} \alpha_{3}, \beta\right)
$$

is a consequence of $\mathfrak{R}$.

(ii) Let $\beta_{1} b_{j} \beta_{2} b_{l} \beta_{3} \in B^{+}$be an arbitrary word of length $m-1 \geq 2$, and let $\alpha \in A^{+}$ be an arbitrary word of length $m$. Then the relation

$$
\xi\left(\alpha, \beta_{1} b_{\theta(j)} b_{\iota(j)} \beta_{2} b_{l} \beta_{3}\right)=\xi\left(\alpha, \beta_{1} b_{j} \beta_{2} b_{\theta(l)} b_{\iota(l)} \beta_{3}\right)
$$

is a consequence of $\mathfrak{R}$. 
Proof. (i) We prove the assertion by induction on $\left|\alpha_{2}\right|$. Consider first the case where $\left|\alpha_{2}\right|=0$. Decompose $\beta$ as $\beta \equiv \beta_{1} \beta_{2} \beta_{3}$, where $\left|\beta_{1}\right|=\left|\alpha_{1}\right|,\left|\beta_{2}\right|=3$ and $\left|\beta_{3}\right|=\left|\alpha_{3}\right|$. Then we have

$$
\begin{aligned}
\xi\left(\alpha_{1} a_{\zeta(i)} a_{\eta(i)} a_{k} \alpha_{3}, \beta\right) & \equiv \xi\left(\alpha_{1}, \beta_{1}\right) \xi\left(a_{\zeta(i)} a_{\eta(i)} a_{k}, \beta_{2}\right) \xi\left(\alpha_{3}, \beta_{3}\right) & & \text { (Lemma 5.8) } \\
& =\xi\left(\alpha_{1}, \beta_{1}\right) \xi\left(a_{i} a_{\zeta(k)} a_{\eta(k)}, \beta_{2}\right) \xi\left(\alpha_{3}, \beta_{3}\right) & & \text { (relation (14)) } \\
& \equiv \xi\left(\alpha_{1} a_{i} \alpha_{2} a_{\zeta(k)} a_{\eta(k)} \alpha_{3}, \beta\right) & & \\
& & & \text { Lemma 5.8) }
\end{aligned}
$$

and the assertion is proved in this case.

Assume inductively that the assertion is true for all words shorter than $n$ for some $n \geq 1$. Let $\alpha_{2} \in A^{+}$be an arbitrary word of length $n$. If we write $\alpha_{2} \equiv a_{m} \alpha_{2}^{\prime}$, we have

$$
\begin{aligned}
& \xi\left(\alpha_{1} a_{\zeta(i)} a_{\eta(i)} \alpha_{2} a_{k} \alpha_{3}, \beta\right) \equiv \xi\left(\alpha_{1} a_{\zeta(i)} a_{\eta(i)} a_{m} \alpha_{2}^{\prime} a_{k} \alpha_{3}, \beta\right) \\
& =\xi\left(\alpha_{1} a_{i} a_{\zeta(m)} a_{\eta(m)} \alpha_{2}^{\prime} a_{k} \alpha_{3}, \beta\right) \quad\left(\text { case }\left|\alpha_{2}\right|=0\right) \\
& =\xi\left(\alpha_{1} a_{i} a_{m} \alpha_{2}^{\prime} a_{\zeta(k)} a_{\eta(k)} \alpha_{3}, \beta\right) \quad \text { (induction) } \\
& \equiv \xi\left(\alpha_{1} a_{i} \alpha_{2} a_{\zeta(k)} a_{\eta(k)} \alpha_{3}, \beta\right) \text {, }
\end{aligned}
$$

and this completes the proof of this part of the lemma.

(ii) The proof is dual to (i), and uses relations (15).

Proof of Proposition 5.7. It is obvious that all the relations of $\mathfrak{R}$ hold in $S \times T$. Thus to complete the proof of the proposition one has to show that any relation $w_{1}=w_{2}$ holding in $S \times T$ is a consequence of $\Re$.

So let $w_{1}, w_{2} \in(A \times B)^{+}$be any two words such that the relation $w_{1}=w_{2}$ holds in $S \times T$. We claim that without loss of generality we may assume that $\left|w_{1}\right|=\left|w_{2}\right|$. Indeed, relations (16) imply that $A \times B$ is a full generating set for the semigroup defined by $\mathfrak{R}$. If $\left|w_{1}\right|<\left|w_{2}\right|$, then Lemma 2.12 implies that there exists a word $w_{1}^{\prime}$ such that $\left|w_{1}^{\prime}\right|=\left|w_{2}\right|$ and the relation $w_{1}=w_{1}^{\prime}$ is a consequence of $\mathfrak{R}$. Now we have that the relation $w_{1}^{\prime}=w_{2}$ holds in $S \times T$ (since all the relations of $\mathfrak{R}$ hold in $S \times T)$, and that $w_{1}=w_{2}$ is a consequence of $\mathfrak{R}$ if and only if $w_{1}^{\prime}=w_{2}$ is a consequence of $\Re$. The case $\left|w_{1}\right|>\left|w_{2}\right|$ is considered analogously, and the claim is proved.

Now let

$$
\begin{aligned}
& w_{1} \equiv\left(a_{i_{1}}, b_{j_{1}}\right)\left(a_{i_{2}}, b_{j_{2}}\right) \ldots\left(a_{i_{p}}, b_{j_{p}}\right), \\
& w_{2} \equiv\left(a_{k_{1}}, b_{l_{1}}\right)\left(a_{k_{2}}, b_{l_{2}}\right) \ldots\left(a_{k_{p}}, b_{l_{p}}\right) .
\end{aligned}
$$

Since the relation $w_{1}=w_{2}$ holds in $S \times T$, the relation

$$
a_{i_{1}} a_{i_{2}} \ldots a_{i_{p}}=a_{k_{1}} a_{k_{2}} \ldots a_{k_{p}}
$$

must hold in $S$. Recall that, by assumption, $S$ has no critical pairs with respect to $\mathfrak{P}$. Thus there exists an elementary sequence

$$
a_{i_{1}} a_{i_{2}} \ldots a_{i_{p}} \equiv \alpha_{1}, \alpha_{2}, \ldots, \alpha_{q} \equiv a_{k_{1}} a_{k_{2}} \ldots a_{k_{p}}
$$

with respect to $\mathfrak{P}$ in which $\left|\alpha_{m}\right| \geq p$ for all $m(1 \leq m \leq q)$.

If we denote the word $b_{j_{1}} b_{j_{2}} \ldots b_{j_{p}}$ by $\beta$, we have the following

Lemma 5.10. For every $m(1 \leq m \leq q)$ the relation

$$
\xi\left(\alpha_{m}, \lambda\left(\beta,\left|\alpha_{m}\right|-p\right)\right)=\xi\left(\alpha_{m+1}, \lambda\left(\beta,\left|\alpha_{m+1}\right|-p\right)\right)
$$

is a consequence of $\mathfrak{R}$. 
Proof. We distinguish three cases, depending on how $\alpha_{m+1}$ is obtained from $\alpha_{m}$.

Case 1. $\alpha_{m} \equiv \alpha_{m+1}$. In this case clearly

$$
\xi\left(\alpha_{m}, \lambda\left(\beta,\left|\alpha_{m}\right|-p\right)\right) \equiv \xi\left(\alpha_{m+1}, \lambda\left(\beta,\left|\alpha_{m+1}\right|-p\right)\right),
$$

and the assertion is true in this case.

Case 2. $\alpha_{m} \equiv \gamma a_{i} \delta, \alpha_{m+1} \equiv \gamma a_{\zeta(i)} a_{\eta(i)} \delta$, with $i \in I$ and $\gamma, \delta \in A^{*}$. If we let

$$
\left|\alpha_{m}\right|-p=r,
$$

then clearly

$$
\left|\alpha_{m+1}\right|-p=r+1 \text {. }
$$

Thus we have

$$
\lambda\left(\beta,\left|\alpha_{m}\right|-p\right) \equiv b_{j_{1}} b_{j_{2}} \ldots b_{j_{p-1}} b_{\theta\left(j_{p}\right)} b_{\theta \iota\left(j_{p}\right)} \ldots b_{\theta \iota^{r-1}\left(j_{p}\right)} b_{\iota^{r}\left(j_{p}\right)},
$$

and

$$
\lambda\left(\beta,\left|\alpha_{m+1}\right|-p\right) \equiv b_{j_{1}} b_{j_{2}} \ldots b_{j_{p-1}} b_{\theta\left(j_{p}\right)} b_{\theta \iota\left(j_{p}\right)} \ldots b_{\theta \iota^{r-1}\left(j_{p}\right)} b_{\theta \iota^{r}\left(j_{p}\right)} b_{\iota^{r+1}\left(j_{p}\right)} .
$$

Let us now decompose the word $\lambda\left(\beta,\left|\alpha_{m}\right|-p\right)$ as

$$
\lambda\left(\beta,\left|\alpha_{m}\right|-p\right) \equiv \beta_{1} b_{j_{l}} \beta_{2},
$$

where, $\left|\beta_{1}\right|=|\gamma|$ and $\left|\beta_{2}\right|=|\delta|$. Now we have

$$
\begin{aligned}
& \xi\left(\alpha_{m}, \lambda\left(\beta,\left|\alpha_{m}\right|-p\right)\right) \\
& \quad \equiv \xi\left(\gamma a_{i} \delta, \beta_{1} b_{j_{l}} \beta_{2}\right) \\
& \quad \equiv \xi\left(\gamma, \beta_{1}\right)\left(a_{i}, b_{j_{l}}\right) \xi\left(\delta, \beta_{2}\right) \\
& \quad=\xi\left(\gamma, \beta_{1}\right)\left(a_{\zeta(i)}, b_{\theta\left(j_{l}\right)}\right)\left(a_{\eta(i)}, b_{\iota\left(j_{l}\right)}\right) \xi\left(\delta, \beta_{2}\right) \\
& \quad \equiv \xi\left(\gamma a_{\zeta(i)} a_{\eta(i)} \delta, \beta_{1} b_{\theta\left(j_{l}\right)} b_{\iota\left(j_{l}\right)} \beta_{2}\right) \\
& \quad \equiv \xi\left(\alpha_{m+1}, \beta_{1} b_{\theta\left(j_{l}\right)} b_{\iota\left(j_{l}\right)} \beta_{2}\right) \\
& \quad \equiv \xi\left(\alpha_{m+1}, b_{j_{1}} b_{j_{2}} \ldots b_{j_{l-1}} b_{\theta\left(j_{l}\right)} b_{\iota\left(j_{l}\right)} b_{j_{l+1}} \ldots b_{\theta_{\iota} r-1\left(j_{p}\right)} b_{\iota^{r}\left(j_{p}\right)}\right) \\
& \quad=\xi\left(\alpha_{m+1}, b_{j_{1}} b_{j_{2}} \ldots b_{j_{l-1}} b_{j_{l}} b_{j_{l+1}} \ldots b_{\theta \iota^{r-1}\left(j_{p}\right)} b_{\iota^{r}\left(j_{p}\right)} b_{\iota^{r+1}\left(j_{p}\right)}\right) \\
& \quad \equiv \xi\left(\alpha_{m+1}, \lambda\left(\beta,\left|\alpha_{m+1}\right|-p\right)\right)
\end{aligned}
$$

as required.

Case 3. $\alpha_{m} \equiv \gamma u \delta, \alpha_{m+1} \equiv \gamma v \delta$, with $(u=v) \in R$ and $\gamma, \delta \in A^{*}$. Recall that we have $|u|=|v|$, since $\mathfrak{P}$ is uniform. Thus we have

$$
\left|\alpha_{m}\right|-p=\left|\alpha_{m+1}\right|-p,
$$

and hence

$$
\lambda\left(\beta,\left|\alpha_{m}\right|-p\right) \equiv \lambda\left(\beta,\left|\alpha_{m+1}\right|-p\right) ;
$$

denote this last word by $\beta^{\prime}$. Decompose $\beta^{\prime}$ as

$$
\beta^{\prime} \equiv \beta_{1}^{\prime} \beta_{2}^{\prime} \beta_{3}^{\prime},
$$

where $\beta_{1}^{\prime}, \beta_{2}^{\prime}, \beta_{3}^{\prime} \in B^{*},\left|\beta_{1}^{\prime}\right|=|\gamma|,\left|\beta_{2}^{\prime}\right|=|u|$ and $\left|\beta_{3}^{\prime}\right|=|\delta|$. Now we have

$$
\begin{aligned}
\xi\left(\alpha_{m}, \lambda\left(\beta,\left|\alpha_{m}\right|-p\right)\right) & \equiv \xi\left(\gamma u \delta, \beta_{1}^{\prime} \beta_{2}^{\prime} \beta_{3}^{\prime}\right) \\
& \equiv \xi\left(\gamma, \beta_{1}^{\prime}\right) \xi\left(u, \beta_{2}^{\prime}\right) \xi\left(\delta, \beta_{3}^{\prime}\right) \\
& =\xi\left(\gamma, \beta_{1}^{\prime}\right) \xi\left(v, \beta_{2}^{\prime}\right) \xi\left(\delta, \beta_{3}^{\prime}\right) \\
& \equiv \xi\left(\gamma v \delta, \beta_{1}^{\prime} \beta_{2}^{\prime} \beta_{3}^{\prime}\right) \\
& \equiv \xi\left(\alpha_{m+1}, \lambda\left(\beta,\left|\alpha_{m+1}\right|-p\right)\right),
\end{aligned}
$$

as required. 
We now continue the proof of Proposition 5.7. As an immediate consequence of Lemma 5.10 we have that the relation

$$
\xi\left(\alpha_{1}, \lambda\left(\beta,\left|\alpha_{1}\right|-p\right)\right)=\xi\left(\alpha_{q}, \lambda\left(\beta,\left|\alpha_{q}\right|-p\right)\right)
$$

is a consequence of $\mathfrak{R}$. Recall that $\alpha_{1} \equiv a_{i_{1}} a_{i_{2}} \ldots a_{i_{p}}$ and $\alpha_{q} \equiv a_{k_{1}} a_{k_{2}} \ldots a_{k_{p}}$, so that in particular $\left|\alpha_{1}\right|=\left|\alpha_{q}\right|=p$. Thus we conclude that the relation

$$
\xi\left(a_{i_{1}} a_{i_{2}} \ldots a_{i_{p}}, b_{j_{1}} b_{j_{2}} \ldots b_{j_{p}}\right)=\xi\left(a_{k_{1}} a_{k_{2}} \ldots a_{k_{p}}, b_{j_{1}} b_{j_{2}} \ldots b_{j_{p}}\right)
$$

is a consequence of $\mathfrak{R}$. A completely analogous argument, based on relations (13), (16) and Lemma 5.9 (i), shows that the relation

$$
\xi\left(a_{k_{1}} a_{k_{2}} \ldots a_{k_{p}}, b_{j_{1}} b_{j_{2}} \ldots b_{j_{p}}\right)=\xi\left(a_{k_{1}} a_{k_{2}} \ldots a_{k_{p}}, b_{l_{1}} b_{l_{2}} \ldots b_{l_{p}}\right)
$$

is a consequence of $\mathfrak{R}$. By combining (20) and (21) we conclude that the relation $w_{1}=w_{2}$ is a consequence of $\Re$, thus completing the proof.

\section{EXAmple: A NON-FINITELY PRESENTED DIRECT PRODUCT}

In this section we construct an example of a semigroup $S$ which is finitely presented and satisfies $S^{2}=S$, but is not stable. As a consequence we obtain examples of finitely presented semigroups $S$ and $T$ such that the direct product $S \times T$ is finitely generated but is not finitely presented.

Theorem 6.1. Let $S$ be the semigroup defined by the presentation

$$
\mathfrak{P}=\left\langle a, x, y \mid x a=a, y a=a, x y=x, y^{2}=y\right\rangle .
$$

Then $S$ satisfies $S^{2}=S$, but $S$ is not stable.

Before we prove the above theorem we establish some properties of $S$.

Lemma 6.2. Let $w_{1}, w_{2} \in\{a, x, y\}^{+}$be any two words such that the relation $w_{1}=$ $w_{2}$ holds in $S$.

(i) The number of occurences of the letter $a$ in $w_{1}$ is equal to the number of occurences of a in $w_{2}$.

(ii) If the last letter in $w_{1}$ is a then the last letter in $w_{2}$ is also a.

(iii) If $w_{1} \in\{x, y\}^{+}$then also $w_{2} \in\{x, y\}^{+}$. Moreover, if the first letter of $w_{1}$ is $x$ then the first letter of $w_{2}$ is $x$ as well.

Proof. (i), (ii) Note that the assertions are true for any relation of $\mathfrak{P}$. The assertions for general $w_{1}$ and $w_{2}$ follow from the fact that $w_{2}$ can be obtained from $w_{1}$ by applying relations from $\mathfrak{P}$.

(iii) The first assertion follows from (i). Thus, the subsemigroup of $S$ generated by $\{x, y\}$ has a presentation $\left\langle x, y \mid x y=x, y^{2}=y\right\rangle$. The second assertion is obviously true for any relation in this presentation. This in turn implies the assertion for arbitrary $w_{1}$ and $w_{2}$ as in (i) and (ii).

Proof of Theorem 6.1. Let us denote the alphabet $\{a, x, y\}$ by $A$. It is clear from the relations that $A \subseteq A^{2}$, i.e. that $A$ is a full generating set for $S$. Thus $S^{2}=S$ by Proposition 2.10 .

It remains to be proved that $S$ is not stable. To this end let

$$
\mathfrak{Q}=\langle A \mid R\rangle
$$


be any finite presentation for $S$ in terms of generators $A$, and let

$$
m=\max \{|u|,|v|:(u=v) \in R\} .
$$

We claim that the pair $\left(x^{m} a, y^{m} a\right)$ is a critical pair for $S$ with respect to $\mathfrak{Q}$. Clearly we have

$$
\left|x^{m} a\right|=\left|y^{m} a\right|=m+1,
$$

and the relation $x^{m} a=y^{m} a(=a)$ holds in $S$. Let us have any elementary sequence

$$
x^{m} a \equiv \alpha_{1}, \alpha_{2}, \ldots, \alpha_{p} \equiv y^{m} a
$$

with respect to $\mathfrak{Q}$. By Lemma 6.2 (i) and (ii) every $\alpha_{j}(1 \leq j \leq p)$ has the form

$$
\alpha_{j} \equiv \alpha_{j}^{\prime} a,
$$

where $\alpha_{j}^{\prime} \in\{x, y\}^{*}$. Let $j$ be the smallest number such that the first letter of $\alpha_{j}$ is $x$ and the first letter of $\alpha_{j+1}$ is distinct from $x$. So we can write

$$
\alpha_{j} \equiv u \beta, \alpha_{j+1} \equiv v \beta,
$$

where $\beta \in A^{*}$ and $u=v$ is a relation from $R$ such that the first letter of $u$ is $x$, and the first letter of $v$ is distinct from $x$. By Lemma 6.2 (iii), $u$ must contain at least one occurence of the letter $a$, and so we must have $\alpha_{j} \equiv u$ and $\beta \equiv \epsilon$. But this implies that

$$
\left|\alpha_{j}\right|=|u| \leq m<m+1=\min \left(\left|x^{m} a\right|,\left|y^{m} a\right|\right),
$$

and so $\left(x^{m} a, y^{m} a\right)$ is indeed a critical pair.

Corollary 6.3. Let $S$ be the semigroup defined by the presentation

$$
\mathfrak{P}=\left\langle a, x, y \mid x a=a, y a=a, x y=x, y^{2}=y\right\rangle,
$$

and let $T$ be any finitely generated infinite semigroup such that $T^{2}=T$. Then the direct product $S \times T$ is finitely generated, but it is not finitely presented regardless of whether or not $T$ is finitely presented.

Proof. The result follows immediately from Theorems 2.1, 3.5 and 6.1.

Remark 6.4. In [3, Theorem 3.1] the authors construct an example of a finitely presented semigroup $S$ which contains a two-sided ideal $T$ such that $T$ is finitely generated (as a semigroup) but is not finitely presented. The results of this paper enable one to construct more such examples. Indeed, let $S$ be a finitely presented semigroup satisfying $S^{2}=S$ which is not stable (e.g. the semigroup defined in Theorem 6.1). Let $S^{1}$ denote the semigroup $S$ with an identity element adjoined to it, and let $T$ be any finitely presented monoid. The semigroup $S^{1} \times T$ is finitely presented as a direct product of two finitely presented monoids. However, the twosided ideal $S \times T$ of $S^{1} \times T$ is not finitely presented by Theorem 3.5, although it is finitely generated by Theorem 2.1 .

\section{Some Classes of StABle SEMigRoups}

In this section we give a sufficient condition for a semigroup to be stable. As a consequence we obtain that the class of all finitely presented infinite semigroups satisfying this sufficient condition has the property that the direct product of any two semigroups from the class is finitely presented. This class contains many well known classes of semigroups, for example finitely presented regular semigroups. 
Definition 7.1. Let $S$ be a semigroup and let $s \in S$. An element $e \in S$ is said to be a relative left (respectively right) identity for $s$ if $e s=s$ (respectively $s e=s$ ).

Theorem 7.2. Let $S$ be a finitely presented semigroup and let $A$ be a finite generating set for $S$. If every element $a \in A$ has a relative left identity $e_{a}$ as well as a relative right identity $f_{a}$, then $S$ is stable.

Proof. Let $\langle A \mid R\rangle$ be any finite presentation for $S$ in terms of $A$. For a word $w \in A^{+}$, let us denote the initial and terminal letters of $w$ by $\iota(w)$ and $\tau(w)$ respectively. Consider the following two finite sets of relations:

$$
\begin{aligned}
& R_{1}=\left\{e_{a} a=a=a f_{a}: a \in A\right\}, \\
& R_{2}=\left\{e_{\iota(v)} u=u=u f_{\tau(v)}:(u=v) \in R \text { or }(v=u) \in R\right\} .
\end{aligned}
$$

It is clear that all the relations from $R_{1} \cup R_{2}$ hold in $S$, and so $S$ has a presentation

$$
\mathfrak{P}=\left\langle A \mid R, R_{1}, R_{2}\right\rangle .
$$

We now prove that $S$ has no critical pairs with respect to $\mathfrak{P}$. To this end we let $w_{1}, w_{2} \in A^{+}$be two arbitrary words such that $w_{1}=w_{2}$ holds in $S$. Since $\langle A \mid R\rangle$ is a presentation for $S$, it follows that there exists an elementary sequence of the form

$$
w_{1} \equiv \alpha_{1}, \alpha_{2}, \ldots, \alpha_{k} \equiv w_{2}
$$

with respect to this presentation. The number

$$
m=\min \left(\left|w_{1}\right|,\left|w_{2}\right|\right)-\min _{1 \leq i \leq k}\left|\alpha_{i}\right|
$$

is certainly non-negative. If $m=0$ then the above elementary sequence shows that $\left(w_{1}, w_{2}\right)$ is not a critical pair for $\mathfrak{P}$. So let us assume that $m>0$.

Let $i(1 \leq i \leq k-1)$ be arbitrary. We claim that there exists an elementary sequence of the form

$$
\alpha_{i} f_{\tau\left(\alpha_{i}\right)}^{m} \equiv \alpha_{i 1}, \alpha_{i 2}, \ldots, \alpha_{i n_{i}} \equiv \alpha_{i+1} f_{\tau\left(\alpha_{i+1}\right)}^{m}
$$

with respect to $\mathfrak{P}$, in which no term is shorter than $\min \left(\left|w_{1}\right|,\left|w_{2}\right|\right)$. The word $\alpha_{i+1}$ can be obtained from $\alpha_{i}$ by one application of one relation from $R$. Hence we may write

$$
\alpha_{i} \equiv \beta u \gamma, \alpha_{i+1} \equiv \beta v \gamma,
$$

for some words $\beta, \gamma \in A^{*}$ and some relation $(u=v) \in R$ or $(v=u) \in R$. If $\gamma \not \equiv \epsilon$ then $\tau\left(\alpha_{i}\right)=\tau\left(\alpha_{i+1}\right)$, and hence the elementary sequence

$$
\alpha_{i} f_{\tau\left(\alpha_{i}\right)}^{m}, \alpha_{i+1} f_{\tau\left(\alpha_{i+1}\right)}^{m}
$$

has the desired property. On the other hand, if $\gamma \equiv \epsilon$ then we have the following elementary sequence:

$$
\begin{array}{ll}
\alpha_{i} f_{\tau\left(\alpha_{i}\right)}^{m} \equiv \beta u f_{\tau(u)}^{m}, & \\
\beta v f_{\tau(u)}^{m}, & \text { (a relation from } R) \\
e_{L(\beta v)}^{m} \beta v f_{\tau(u)}^{m}, & \text { (a relation from } R_{1}, m \text { times) } \\
e_{L(\beta v)}^{m} \beta v, & \text { (a relation from } R_{2}, m \text { times) } \\
e_{\iota(\beta v)}^{m} \beta v f_{\tau(v)}^{m}, & \text { (a relation from } R_{1}, m \text { times) } \\
\beta v f_{\tau(v)}^{m} \equiv \alpha_{i+1} f_{\tau\left(\alpha_{i+1}\right)}^{m}, & \text { (a relation from } R_{1}, m \text { times) }
\end{array}
$$

which also has the desired property. 
Now, if we combine elementary sequences (22) for all $i(1 \leq i \leq k-1)$ together with elementary sequences

$$
\begin{aligned}
& w_{1} \equiv \alpha_{1}, \alpha_{1} f_{\tau\left(\alpha_{1}\right)}, \alpha_{1} f_{\tau\left(\alpha_{1}\right)}^{2}, \ldots, \alpha_{1} f_{\tau\left(\alpha_{1}\right)}^{m}, \\
& \alpha_{k} f_{\tau\left(\alpha_{k}\right)}^{m}, \alpha_{k} f_{\tau\left(\alpha_{k}\right)}^{m-1}, \ldots, \alpha_{k} f_{\tau\left(\alpha_{k}\right)}, \alpha_{k} \equiv w_{2},
\end{aligned}
$$

arising from multiple applications of relations from $R_{1}$, we obtain an elementary sequence from $w_{1}$ to $w_{2}$ with respect to $\mathfrak{P}$ in which no term is shorter than $\min \left(\left|w_{1}\right|,\left|w_{2}\right|\right)$.

We have proved that $S$ has no critical pairs with respect to $\mathfrak{P}$. Therefore, $S$ is stable as required.

Corollary 7.3. Let $S$ and $T$ be two finitely presented infinite semigroups both satisfying the conditions of Theorem 7.2. Then the direct product $S \times T$ is finitely presented.

Proof. By Theorem 7.2 both $S$ and $T$ are stable. Moreover, from $a=a f_{a}$ for each generator $a$ of $S$, it is easy to see that $S^{2}=S$, and similarly $T^{2}=T$. Therefore $S \times T$ is finitely presented by Theorem 3.5 .

Remark 7.4. The conditions of Theorem 7.2 are satisfied by all finitely presented semigroups $S=\langle A \mid R\rangle$ belonging to various well known classes of semigroups. First of all, if $S$ is any finitely presented monoid, then, clearly, every element has a relative right identity and a relative left identity, namely the identity of $S$. This, of course, includes the case of groups. Next, every element in a regular semigroup has relative left and right identities. Recall that a semigroup $S$ is regular if for every element $s \in S$ there exists an element $s^{\prime} \in S$ such that $s s^{\prime} s=s$. Thus the elements $s s^{\prime}$ and $s^{\prime} s$ are respectively relative left and relative right identities for $s$. This includes the case of $S$ being an inverse semigroup, or a completely (0-)simple semigroup, or a union of groups. (For definitions of various types of semigroups, see [9].) Finally, if $S$ is a finitely generated commutative monoid satisfying $S^{2}=S$, it satisfies the conditions of the theorem. First, by Rédei's theorem [17] $S$ is finitely presented; see also [5] and [6]. Then, if we assume (without loss of generality) that $A$ is an irredundant generating set of $S$, from $S^{2}=S$ we obtain $a=w_{a} a=a w_{a}$ for some non-empty word $w_{a}$.

Remark 7.5. The condition of having relative left and right identities is not necessary for a semigroup to be stable. Indeed, if $S$ is the semigroup defined by

$$
\left\langle a, b \mid a^{2}=a, b a=b\right\rangle,
$$

then it is easily seen to be stable and satisfy $S^{2}=S$, but the element $b$ has no relative left identity. On the other hand, the theorem is no longer valid if we just assume that every generator of $S$ has (say) a relative left identity; one counterexample is provided by the semigroup given in Theorem 6.1. However, if $S$ has a (global) left identity, then the proof of Theorem 7.2 can be easily modified to prove that $S$ is again stable.

\section{Direct PRoducts of one Finite AND ONE INFinite SEMigroup}

The two main results proved so far (Theorems 2.1 and 3.5) give necessary and sufficient conditions for the direct product of two infinite semigroups to be finitely generated or finitely presented. The technical results developed in order to prove 
these theorems can also be used in the case of direct products of one finite and one infinite semigroup.

In addition, in this section we shall make use of some results about subsemigroups of finite index. If $S$ is a semigroup and $T$ is a subsemigroup of $S$, then the (Rees) index of $T$ in $S$ is the number $|S \backslash T|$. This notion of index was first introduced by Jura [12, 13]; see also [22]. Although this index is not a generalisation of the well known index from group theory, they have many properties in common. In particular we have the following Reidemeister-Schreier type result:

Proposition 8.1. Let $S$ be a semigroup and let $T$ be a subsemigroup of finite index in $S$. Then

(i) $S$ is finitely generated if and only if $T$ is finitely generated;

(ii) $S$ is finitely presented if and only if $T$ is finitely presented.

The first result was proved in [12] and reproved in [1]. The second result is from [21]; see also [2].

For a semigroup $S$ we denote by $S^{1}$ the semigroup obtained by adjoining an identity to $S$ if $S$ does not already have one. Clearly, $S$ has finite index in $S^{1}$. Also, since $S^{1}$ is a monoid, it satisfies $\left(S^{1}\right)^{2}=S^{1}$.

Now we have

Theorem 8.2. Let $S$ be a finite semigroup and let $T$ be an infinite semigroup. Then the direct product $S \times T$ is finitely generated if and only if $S^{2}=S$ and $T$ is finitely generated.

Proof. Assume that $S \times T$ is finitely generated. Then we must have $S^{2}=S$ by Lemma 2.3. Also, $T$ must be finitely generated by Lemma 2.2 .

Conversely, assume that $S^{2}=S$ and that $T$ is finitely generated. Then $T^{1}$ must also be finitely generated by Proposition 8.1. Since $T^{1}$ is a monoid it satisfies $\left(T^{1}\right)^{2}=T^{1}$, and hence $S \times T^{1}$ is finitely generated by Proposition 2.5. Finally we note that $S \times T$ has finite index in $S \times T^{1}$, and hence is finitely generated by Proposition 8.1.

We also have the following criterion for finite presentability:

Theorem 8.3. Let $S$ be a finite semigroup and let $T$ be an infinite semigroup. Then the direct product $S \times T$ is finitely presented if and only if the following three conditions are satisfied:

(i) $S^{2}=S$;

(ii) $S$ is stable;

(iii) $T$ is finitely presented.

Proof. We first claim that, to prove the theorem, it is enough to consider the case where $T$ is a monoid. Indeed, the semigroup $S \times T^{1}$ contains $S \times T$ as a subsemigroup of finite index, and hence $S \times T$ is finitely presented if and only if $S \times T^{1}$ is finitely presented by Proposition 8.1 (ii).

So let us assume that $T$ is a monoid. In particular we have $T^{2}=T$. Now the direct part follows from Theorem 8.2 and Proposition 4.1, while the converse part follows from Proposition 5.7.

Example 8.4. Let $S$ be the semigroup defined by the presentation

$$
\left\langle a, x, y \mid x a=a, y a=a, x y=x, a^{3}=a^{2}, x^{2}=x, y^{2}=y\right\rangle .
$$


Obviously, this semigroup is a homomorphic image of the semigroup defined in Theorem 6.1. Actually, the proof of Theorem 6.1 also proves that $S$ is not stable, as the additional relations do not affect the argument. However, $S$ is finite. Indeed, it has 11 elements, as can be verified computationally by using the Todd-Coxeter enumeration procedure (see [24], [15], [11], [18], [25]) or directly. Thus, if $T$ is any finitely generated infinite semigroup, then $S \times T$ is finitely generated by Theorem 8.2 , but is not finitely presented by Theorem 8.3 .

\section{REFERENCES}

[1] C.M. Campbell, E.F. Robertson, N. Ruškuc and R.M. Thomas, Reidemeister-Schreier type rewriting for semigroups, Semigroup Forum 51 (1995), 47-62. MR 96f:20094

[2] C.M. Campbell, E.F. Robertson, N. Ruškuc and R.M. Thomas, On subsemigroups of finitely presented semigroups, J. Algebra 180 (1996), 1-21. CMP 96:08

[3] C.M. Campbell, E.F. Robertson, N. Ruškuc and R.M. Thomas, Presentations for subsemigroups - applications to ideals of semigroups, J. Pure Appl. Algebra, to appear.

[4] C.M. Campbell, E.F. Robertson, N. Ruškuc and R.M. Thomas, On subsemigroups and ideals in free products of semigroups, Internat. J. Algebra Comput. 6 (1996), 571-591. MR 97g:20072

[5] K. Corradi and S. Szabo, A new proof of Redei's theorem, Pacific J. Math. 140 (1989), 53-61. MR 90i:20056

[6] P.A. Grillet, A short proof of Redei's theorem, Semigroup Forum 46 (1993), 126-127. CMP 93:04

[7] P. Hall, The eulerian functions of a group, Quart. J. Math. 7 (1936), 134-151.

[8] P.M. Higgins, Techniques of Semigroup Theory, Oxford University Press, Oxford, 1992. MR 93d:20101

[9] J.M. Howie, Fundamentals of Semigroup Theory, Clarendon Press, Oxford, 1995.

[10] J.M. Howie and N. Ruškuc, Constructions and presentations for monoids, Comm. Algebra 22 (1994), 6209-6224. MR 95k:20092

[11] A. Jura, Coset enumeration in a finitely presented semigroup, Canad. Math. Bull. 21 (1978), 37-46. MR 58:5994

[12] A. Jura, Determining ideals of a given finite index in a finitely presented semigroup, Demonstratio Math. 11 (1978), 813-827. MR 80d:20057

[13] A. Jura, Some remarks on non-existence of an algorithm for finding all ideals of a given index in a finitely presented semigroup, Demonstratio Math. 13 (1980), 573-578. MR 82f:20083

[14] W. Magnus, A. Karrass and D. Solitar, Combinatorial Group Theory, Interscience Publishers, New York, 1966. MR 34:7617

[15] B.H. Neumann, Some remarks on semigroup presentations, Canad. J. Math. 19 (1967), 1018-1026; 20 (1968), 511. MR 36:2677, 5703

[16] S.J. Pride, Geometric methods in combinatorial semigroup theory, Semigroups, Formal Languages and Groups, J. Fountain (ed.), Kluwer, Dordrecht, 1995, pp. 215-232.

[17] L. Rédei, The Theory of Finitely Generated Commutative Semigroups, Pergamon Press, Oxford, 1965. MR 32:5761

[18] E.F. Robertson and Y. Ünlü, On semigroup presentations, Proc. Edinburgh Math. Soc. 36 (1993), 55-68. MR 94a:20100

[19] N. Ruškuc, Matrix semigroups - generators and relations, Semigroup Forum 51 (1995), 319333. MR 96k:20119

[20] N. Ruškuc, Semigroup Presentations, Ph.D. Thesis, University of St Andrews, St Andrews, Scotland, 1995.

[21] N. Ruškuc, On large subsemigroups and finiteness conditions of semigroups, Proc. London Math. Soc., to appear.

[22] N. Ruškuc and R.M. Thomas, Syntactic and Rees indices of subsemigroups, submitted.

[23] C.C. Sims, Computation With Finitely Presented Groups, Cambridge University Press, Cambridge, 1994. MR 95f:20053

[24] J.A. Todd and H.S.M. Coxeter, A practical method for enumerating the cosets of a finite abstract group, Proc. Edinburgh Math. Soc. 5 (1936), 26-34. 
[25] T.G. Walker, Semigroup Enumeration - Computer Implementation and Applications, Ph.D. Thesis, University of St Andrews, St Andrews, Scotland, 1992.

[26] J. Wiegold, Growth sequences of finite groups III, J. Austral. Math. Soc. 25 A (1978), 142144. MR 80c: 20028

Mathematical Institute, University of St Andrews, St Andrews KY16 9SS, Scotland E-mail address: efr@st-and.ac.uk

Mathematical Institute, University of St Andrews, St Andrews KY16 9SS, Scotland

E-mail address: nr1@st-and.ac.uk

School of Mathematics, University of Wales, College of Cardiff, Senghenydd Road, Cardiff, CF2 4AG, Wales

E-mail address: SMAJW@cardiff.ac.uk 